\title{
Disruptores endocrinos y su camino hacia el desequilibrio metabólico
}

\section{Endocrine disruptors and their pathway to metabolic imbalance}

\author{
Sánchez $P^{1,2,3}$, Zanabria $M^{2}$, Latorre $S^{2}$, Calvache $J^{2}$, Coy $A 1,{ }^{2}$, Rojas $W 1,{ }^{2}$
}

${ }^{1}$ Médico especialista en Endocrinología, Fundación Universitaria de Ciencias de la Salud.

${ }^{2}$ Médico especialista en Medicina Interna.

${ }^{3}$ Endocrinólogo Clínica Las Américas, Medellín, Colombia. Grupo COD2.

Autor de correspondencia: Pedro Sánchez Márquez

Correo electrónico: pedros0822@hotmail.com

Fecha de recepción: 25/06/2019

Fecha de aceptación: 30/10/2019

\section{Resumen}

El presente artículo de revisión tiene como objetivo presentar, de forma resumida, la evidencia que existe sobre las repercusiones metabólicas a nivel de obesidad y diabetes, que se genera como consecuencia de la exposición a sustancias químicas exógenas, denominadas disruptores endocrinos (DE), a las cuales nos exponemos de forma cotidiana y que afectan nuestra salud y la de nuestra descendencia. Adicionalmente, con la presente revisión hacemos un llamado no solo a la comunidad médica, sino a los sectores involucrados en la producción, distribución y reglamentación del uso de estas sustancias, pues cada vez hay más evidencia de los efectos nocivos que pueden generar y debemos evitar su uso.

Los datos se obtuvieron de estudios clínicos aleatorizados y de una revisión en idioma español e inglés de los últimos 15 años, que incluyó los términos DeCS: disruptores endocrinos, con alternativa DeCS: sustancias disruptoras endocrinas y efecto disruptor endocrino, así como términos MeSH: endocrine disruptors y alternativas MeSH: disruptors, endocrine; endocrine disrupting chemicals; chemicals, endocrine disrupting; endocrine disruptor effect; disruptor effect, endocrine; effect, endocrine disruptor; endocrine disruptor effects; disruptor effects, endocrine; effects, endocrine disruptor.

Palabras clave: disruptores endocrinos, diabetes mellitus tipo 1, diabetes mellitus tipo 2, dibutil ftalato, fitoestrógenos, obesidad, parabenos.

\begin{abstract}
Since the last century, lifestyle changes have made us increasingly dependent on industrial tools for our current days duties. Without realizing it, many of these components can be harmful to our health, it is the case of chemical products which in animal and human studies have shown alterations of the immune system, increasing the risk of endocrinopathies such as type 1 diabetes (Dt1), type 2 diabetes (Dt2) and obesity; these components are known as endocrine disruptors (ED); The objective of this narrative description is to mention the more frequently used ED associated with the endocrine pathologies.
\end{abstract}

Keywords: Endocrine disruptors, diabetes mellitus, type 1, diabetes mellitus, type 2, dibutyl phthalate, obesity, parabens, phytoestrogens.

El sistema endocrino regula el metabolismo de carbohidratos, grasas y proteínas, lo que asegura el adecuado aporte energético a las necesidades tisulares. Este control depende de un equilibrio mediado por hormonas, las cuales mantienen niveles constantes de glucosa en sangre, principalmente por la producción hepática y renal de glucosa y los depósitos de grasa en el tejido adiposo.

Con los estilos de vida del siglo XXI, nos encontramos expuestos a un medio ambiente hostil para nuestra salud. Desde la década de 1990 se han descrito químicos que interrumpen el delicado equilibrio metabólico, denominados disruptores endocrinos (DE), los cuales son sustancias exógenas que causan efectos adversos sobre la salud de un organismo intacto y su descendencia, con cambios en la función endocrina, estos interfieren con la síntesis, secreción, transporte, sitio de acción y metabolismo de diversas hormonas endocrinas $(1,2)$. Estas sustancias se encuentran como compuestos altamente heterogéneos, que incluyen solventes, compuestos plásticos, pesticidas e incluso algunos agentes farmacéuticos. Su exposición se ha asociado con diversas patologías, como el síndrome metabólico, diabetes, enfermedades cardiovasculares y cáncer. Dependiendo del momento de exposición, estos pueden afec- 
tar a la persona expuesta y su descendencia por un mecanismo propuesto como la hipótesis del desarrollo de los orígenes de la salud y la enfermedad. Según el proceso alterado reciben diferentes términos, como el de obesógenos, cuando alteran el control de la función adipocitaria.

\section{Obesógenos}

Se encuentran presentes en agentes industriales, farmacéuticos, agropecuarios, plásticos, preservantes y pesticidas; estos pueden interferir en la homeostasis del tejido adiposo. El tiempo de exposición es proporcional a las consecuencias asociadas, si la exposición es in utero o en estados tempranos posnatales se aumenta permanentemente el número de adipocitos en la vida adulta, lo que predispondrá a mayor acumulación de otros DE, pues, al ser muchos de ellos lipofílicos, estos crean un círculo vicioso al acumularse en este tejido, lo que estimula su aumento y la acumulación de más DE, que adicionalmente tiene efectos nocivos, como es el caso de los xenobióticos, que forman conjugados de ácidos grasos con efectos tóxicos locales.

Por un lado, estos llevan a un aumento del número y tamaño de los adipocitos, con alteración en el metabolismo lipídico, mediante la interferencia de los reguladores transcripcionales, como los receptores activados de proliferadores de peroxisomas (PPAR) alfa $(\alpha)$, delta $(\delta)$ y gama $(\gamma)(3)$. El PPAR $\gamma$ favorece la diferenciación de los progenitores adipocitarios, de preadipocitos a adipocitos y regula la biosíntesis y almacenamiento lipídico, por ejemplo, los ftalatos y parabenos presentes en las botellas y latas de bebidas, así como el revestimiento de tuberías y selladores dentales son potentes activadores del PPAR $\gamma$, lo que promueve la diferenciación de células mesenquimales en adipocitos (4), por su parte, el PPAR $\alpha$ estimula la beta-oxidación de ácidos grasos. Adicionalmente pueden interferir sobre los receptores esteroideos, lo que altera el almacenamiento lipídico y el depósito de tejido graso. Dentro de estos, los fitoestrógenos, como la genisteína y la daidzeína, presentes en la soya y legumbres, han mostrado, en modelos murinos y en mujeres posmenopáusicas, que llevan a acumulación de grasa $(5,6)$. Asimismo, la utilización de fitoestrógenos en roedores durante la gestación o lactancia contribuye a obesidad en la descendencia (7).

En este mismo sentido se ha encontrado una relación entre la exposición a productos perfluoro alquilados, en especial ácido perfluorooctanoico en la niñez y al aumento de adiposidad en la adolescencia y la edad adulta temprana (8). Adicionalmente, al incrementar el tejido adiposo disminuye la sensibilidad a la insulina y se altera el metabolismo lipídico. Por otra parte, también se regula la sensación de hambre/saciedad, lo que altera el balance energético a favor de un almacenamiento calórico. Se ha observado una correlación entre los niveles de bisfenol A (BPA) y leptina/ghrelina en modelos in vitro de células adipocitarias, que aumentan la producción de leptina luego de la exposición a BPA y en modelos murinos de posexposición a metilparabenos (9). En pacientes sometidos a cambios de dieta para pérdida de peso se ha observado que la exposición a productos con perfluoro alquilados (ácido perfluoro octanosulfónico y ácido perfluoro octanoico) se asocia con ganancia de peso, especialmente en mujeres, esto se relacionó con disminución de la tasa metabólica basal (10).

En tejido adiposo pardo, la exposición a contaminantes ambientales, como el dicloro-difenil-tricloroetano (DDT), y su metabolito dicloro-difenil-etileno (DDE), se asocian con un mayor riesgo de obesidad, que podría relacionarse con menor actividad del tejido adiposo pardo (11).

\section{Diabetes tipo 1 (DM1)}

Los DE pueden afectar glándulas como el páncreas (12) pues se han asociado con alteraciones del desarrollo tanto del sistema inmune como de las células pancreáticas. Su exposición durante el desarrollo fetal o en edades tempranas puede asociarse con diabetes mellitus tipo 1, como se ha evidenciado en mujeres expuestas a altos niveles de contaminación y en modelos animales (13-15). Aquellas personas expuestas a altos niveles de bisfenol A (BPA) y ácido perfluoro undecanoico aceleraron el proceso de insulitis y desarrollo de diabetes, aunque con resultados contradictorios con otros estudios en probable relación con el tiempo y grado de exposición $(16,17)$.

Estudios epidemiológicos han observado que la exposición in utero a arsénico se asocia a cambios inmunológicos en recién nacidos, los cuales se relacionan con el desarrollo de DM1 (18). Estudios experimentales in vitro desarrollados en modelos animales han asociado la exposición prenatal a tetraclorobidenzo p dioxina (TCDD) con autoinmunidad posnatal (19).

En las células $\beta$ pancreáticas, los DE aumentan la sensibilidad al daño por parte del sistema inmune, llevando a apoptosis y perpetuando la autoinmunidad al producir péptidos específicos contra las células $\beta$ (20). Por otra parte, la alteración en la homeostasis del calcio y el estrés sobre el retículo endoplásmico en las células beta pancreáticas produce apoptosis celular, lo que afecta los islotes pancreáticos con ineficiencia en la secreción de insulina, lo cual se ha demostrado en estudios longitudinales que muestran cambios en los niveles de insulina en niños y adolescentes $(21,22)$. En modelos animales, la exposición a BPA, ftalatos, arsénico y bifenilos in utero lleva a pérdida de la masa de células beta y producción de insulina $(23,24)$. Asimismo, se ha comprobado que el BPA altera la función y el número de macrófagos y linfocitos B, además de causar una importante desregulación en la producción de una gran variedad de citocinas, siendo la más relevante una disminución en la secreción de IL-6, IL-10 e interferón gamma, en asociación con la génesis de la diabetes tipo 1 (25). 


\section{Hay órganos que sufren y no se ven ${ }^{1}$ La diabetes es más que glucosa ${ }^{1}$}

\section{Uso Temprano}

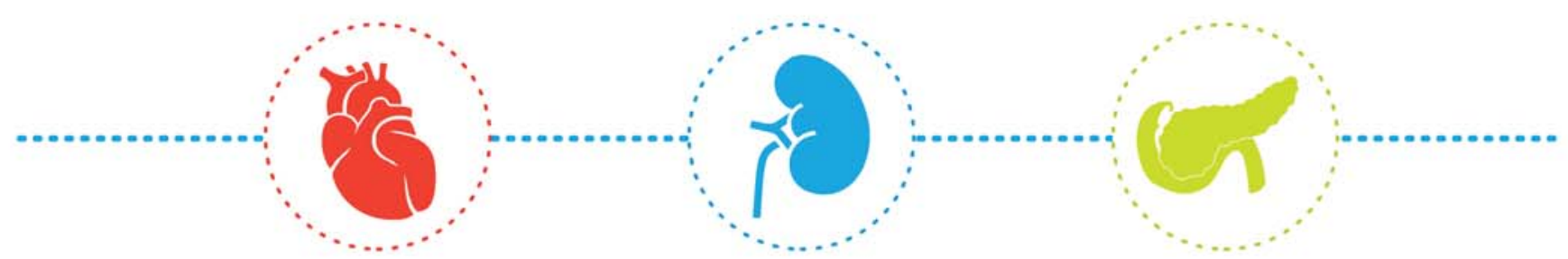

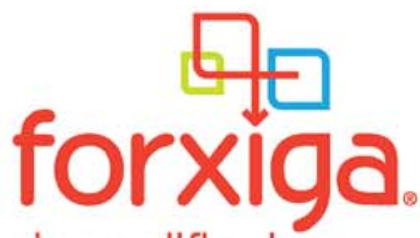

dapagliflozina

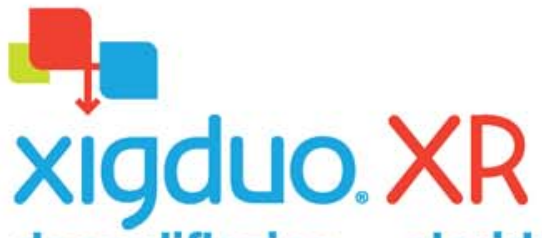

dapagliflozina + cloridrato de metformina

\section{EFICACIA: $\downarrow H b A 1 c$, Peso y PA28}

\section{AMPLIO PERFIL DE SEGURIDAD2-3}

\section{CONCIENCIA CARDIORRENAL2-3,9}

Esto puede ocurrir de forma temprana, incluso antes de que su paciente sea dignosticado ${ }^{10}$ ¿Está su paciente con DMT2 protegido?

HbA1c: hemoglobina glucosilada; PA: Presión Arterial; DMT2: diabetes mellitus tipo 2.

Referencias: 1. Chen-Ku CH, et al. Vascular complications in patients with type 2 diabetes: prevalence and comorbidities in 6 countries of Latin America (a cohort type 2 diabetes who have igam). Endocr Pract. 2019 Oct; 25(10):994-1002. 2. IPP Forxiga. 3. IPP Xigduo XR. 4. Bailey, et al. Effect of dapagliflozin in patients with 5. Ferrannini E, et al. Diabetes Care 2010;33:2217-24. 6. Bolinder J. Diabetes Obes Metab. 2014 Fob;16(2):159-69. 7. Rosenstock, et al. Composite Endpoint Analysis of Dapagliflozin versus Saxagliptin as Add-On Therapy in Patients with Type 2 Diabetes Inadequately Controlled with Metformin. Presented at the 14th Rosenstock J et al, Arch Endocrinol Metab 2018; 62(4):424-430.9. Sonesson, et al. Cardiovascular effects, of dapaglifiozin in patients with type 2 diabetes. and different risk categories: a meta-analysis. Cardiovasc Diabetol (2016) 15:37 10. Gedebjerg, et al. Prevalence of micro- and macrovascular diabetes complication at time of T2D diagnosis and associated clinical characteristics. J Diabetes Complications. 2018 Jan;32(1):34-40. 
En estudios genéticos se ha demostrado que el BPA actúa como antagonista del receptor de andrógenos, lo que altera su función. En la actualidad, existe cada vez más evidencia del papel que desempeñan los andrógenos en la diabetes mellitus. Por ejemplo, se ha visto que la deficiencia de testosterona aumenta el riesgo de síndrome metabólico, diabetes mellitus y enfermedades vasculares. Por ende, esta es otra de las vías que explican la asociación positiva entre la BPA y el desarrollo de diabetes mellitus (26). Adicionalmente se ha observado que los DE alteran la permeabilidad, inflamación y microbiota intestinal, lo cual se asocia con el desarrollo de DM1.

\section{Diabetes tipo 2}

Se ha descrito que los DE interfieren con la secreción y acción de la insulina, el metabolismo de la glucosa, como consecuencia, favorece el desarrollo de la diabetes mellitus tipo 2. Dentro de estos se encuentran relacionados los bifenilos policlorados, organoclorados, pesticidas, BPA y ftalatos, entre otros (27). La relación causal se ha propuesto por estudios observacionales que han documentado, por ejemplo, cómo la proximidad residencial con lugares de desecho contaminado por bifenilos policlorados se asocia con mayores tazas de hospitalización por diabetes, incluso se han realizado mediciones séricas, en las que se encontró una asociación significativa entre su concentración y la presencia de diabetes mellitus.

En una revisión sistemática de la literatura que incluyó 41 estudios de corte transversal y 8 estudios prospectivos y en donde se realizaron mediciones séricas y urinarias de algunos disruptores endocrinos, Song y colaboradores encontraron una relación de riesgo entre dioxinas, bifenilo policlorado y pesticidas clorinatados con la diabetes mellitus tipo 2 (28). Se ha observado aumento de los niveles de glucosa sérica en ayunas y posprandial, así como de niveles de insulina basal $\mathrm{y}$, con esto, mayor riesgo de diabetes mellitus en las mujeres expuestas a bifenilo policlorado; sin embargo, otros estudios muestran que la asociación de la exposición a bisfenol A y el desarrollo de diabetes es positiva únicamente en individuos genéticamente predispuestos para el desarrollo de diabetes mellitus tipo 2 con un OR de 1,89, mientras que no se demostró la asociación en aquellos sin predisposición genética (29).
En la actualidad, la utilización frecuente de pesticidas se ha asociado con un aumento del riesgo de diabetes mellitus, predominantemente en poblaciones cercanas a los cultivos que utilizan dichos pesticidas, al ser más probable encontrar presencia de los mismos en el agua y diferentes alimentos; para que este riesgo sea considerable se requiere una exposición continua por varios años. Los organoclorados y las dioxinas son los dos tipos de pesticidas que se han visto asociados con dicho riesgo. La aldrina, dieldrina y heptacloro (organoclorados), así como el ácido triclorofenoxiacético (dioxina), son los productos que se asocian con mayor riesgo de diabetes mellitus, ya que se ha demostrado que alteran el metabolismo de la glucosa, lo que resulta en elevación en la concentración sérica de la glucosa e insulina (26).

\section{Conclusiones}

Las enfermedades crónicas no transmisibles son la pandemia del siglo XXI, en la actualidad, nos encontramos expuestos a un ambiente hostil que vulnera nuestra salud. Los agentes químicos, denominados disruptores endocrinos, han mostrado afectar la homeostasis metabólica, lo que lleva a patologías como obesidad y diabetes tipo 1 y 2 , no solo de las personas expuestas, sino también su descendencia, con el uso creciente de estos agentes en diferentes industrias, como los pesticidas y la fabricación de elementos de uso diario, como los plásticos. El personal de salud y las entidades regulatorias deben estar al tanto de los efectos nocivos que estos agentes pueden generar y tomar así las medidas pertinentes para la prevención de las enfermedades asociadas o, una vez que estas se presentan, realizar el diagnóstico oportuno para dar el tratamiento apropiado y evitar sus complicaciones.

\section{Conflictos de interés}

Ninguno

\section{Fuentes de financiación}

Ninguna. 


\section{Referencias}

1. Bergman Å, Heindel J, Kasten T, Kidd K, Jobling S, Neira M, et al. The impact of endocrine disruption: a consensus statement on the state of the science. Environ Health Perspect. 2013;121(4):A104-6.

2. Zoeller R, Brown T, Doan L, Gore A, Skakkebaek N, Soto A, et al. Endocrinedisrupting chemicals and public health protection: a statement of principles from the Endocrine Society. Endocrinology. 2012;153(9):4097-110.

3. Ferre P. The biology of peroxisome proliferator-activated receptors. Diabetes. 2004;53(1):S43-50.

4. Janesick A, Blumberg B. Minireview: PPAR $\gamma$ as the target of obesogens. J Steroid Biochem Mol Biol. 2011;127(1-2):4-8.

5. Kim HK, Nelson-Dooley C, Della-Fera M, Yang JY, Zhang W, Duan J, et al. Genistein decreases food intake, body weight, and fat pad weight and causes adipose tissue apoptosis in ovariectomized female mice. J Nutr. 2006;136(2):409-14.

6. Wu J, Oka J, Tabata I, Higuchi M, Toda T, Fuku N, et al. Effects of isoflavone and exercise on BMD and fat mass in postmenopausal Japanese women: a 1-year randomized placebo-controlled trial. J Bone Miner Res. 2006.

7. Ruhlen R, Howdeshell K, Mao J, Taylor J, Bronson F, Newbold R, et al. Low phytoestrogen levels in feed increase fetal serum estradiol resulting in the "fetal estrogenization syndrome" and obesity in CD-1 mice. Environ Health Perspect. 2008;116;3:322-8.

8. Domazet SL, Grontved A, Timmermann AG, Nielsen F, Jensen TK. Longitudinal associations of exposure to perfluoroalkylated substances in childhood and adolescence and indicators of adiposity and glucose metabolism 6 and 12 years later: the European youth heart study. Diabetes Care. 2016;39(10):1745-51.

9. Rönn M, Lind L, Örberg J, Kullberg J, Söderberg S, Larsson A, et al. Bisphenol A is related to circulating levels of adiponectin, leptin and ghrelin, but not to fat mass or fat distribution in humans. Chemosphere. 2014;112:42-8.

10. Liu G, Dhana K, Furtado J, Rood J, Zong G, Liang L, et al. Perfluoroalkyl substances and changes in body weight and resting metabolic rate in response to weight-loss diets: a prospective study. PLoS Med. 2018;13;15(2):e1002502.

11. Paolella G, Burgos Aceves M, Lionetti L, Di Gregorio I, Busiello R, Lepretti M. Environmental pollutants effect on brown adipose tissue. Front Physiol. 2019;9:1-8.

12. Gore A, Chappell V, Fenton S, Flaws J, Nadal A, Prins G, et al. The endocrine society's second scientific statement on endocrine-disrupting chemicals. Endocr Rev. 2015;36(6):E1-E150.

13. Bodin J, Stene L, Nygaard U. Can exposure to environmental chemicals increase the risk of diabetes type 1 development? Biomed Res Int. 2015;208947:19.

14. Howard S, Lee D. What is the role of human contamination by environmental chemicals in the development of type 1 diabetes? J Epidemiol Community Health. 2012;66(6):479-81.
15. Eze I, Hemkens L, Bucher H, Hoffmann B, Schindler C, Künzli N, et al. Association between ambient air pollution and diabetes mellitus in Europe and North America: systematic review and meta-analysis. Environ Health Perspect. 2015;123(5):381-9.

16. Bodin J, Bølling A, Becher R, Kuper F, Løvik M, Nygaard U. Transmaterna bisphenol an exposure accelerates diabetes type 1 development in NOD mice. Toxicol Sci. 2014;137(2):311-23.

17. Bodin J, Groeng E, Andreassen M, Dirven H, Nygaard U. Exposure to perfluoroundecanoic acid (PFUnDA) accelerates insulitis development in a mouse model of type 1 diabetes. Toxicol Reports. 2016; 29;3:664-72.

18. Grau-Pérez M, Kuo C, Spratlen M, Thayer K, Mendez M, Hamman R, et al. The association of arsenic exposure and metabolism with type 1 and type 2 diabetes in youth: the search case-control study. Diabetes Care. 2017;40(1):46-53.

19. Xu J, Huang G, Guo T. Developmental bisphenol A exposure modulates immune-related diseases. Toxics. 2016;26;4(4).

20. Dahlquist G. Can we slow the rising incidence of childhood-onset autoimmune diabetes? The overload hypothesis. Diabetologia. 2006;49(1):20-4.

21. Ahn C, Kang H, Lee J, Hong E, Jung E, Yoo Y, et al. Bisphenol A and octylphenol exacerbate type 1 diabetes mellitus by disrupting calcium homeostasis in mouse pancreas. Toxicol Lett. 2018;295:162-72.

22. Debost-Legrand A, Warembourg C, Massart C, Chevrier C, Bonvallot N Monfort C, et al. Prenatal exposure to persistent organic pollutants and organophosphate pesticides, and markers of glucose metabolism at birth. Environ Res. 2016;146:207-17.

23. Sant K, Jacobs H, Borofski K, Moss J, Timme-Laragy A. Embryonic exposures to perfluorooctanesulfonic acid (PFOS) disrupt pancreatic organogenesis in the zebrafish, Danio rerio. Environ Pollut. 2017;220(B):807-17.

24. Sant K, Jacobs H, Xu J, Borofski K, Moss L, Moss J, et al. Assessment of toxicological perturbations and variants of pancreatic islet development in the zebrafish model. Toxics. 2016;4(3): pii: 20 .

25. Bodin J, Kocbach Bølling A, Wendt A, Eliasson L, Becher R, Kuper F, et al Exposure to bisphenol A, but not phthalates, increases spontaneous diabetes type 1 development in NOD mice. Toxicol Reports. 2015;2:99-110.

26. Sakkiah S, Wang T, Zou W, Wang Y, Pan B, Tong W, et al. Endocrine disrupting chemicals mediated through binding androgen receptor are associated with diabetes mellitus. Int J Environ Res Public Health. 2017;15(1):25

27. Ruiz D, Becerra M, Jagai J, Ard K, Sargis R. Disparities in environmental exposures to endocrine-disrupting chemicals and diabetes risk in vulnerable populations. Diabetes Care. 2018;41(1):193-205.

28. Song Y, Chou EL, Baecker A, You NCY, Song Y, Sun Q, et al. Endocrine-disrupting chemicals, risk of type 2 diabetes, and diabetes-related metabolic traits: a systematic review and meta-analysis. J Diabetes. 2016;8(4):516-32.

29. Bi Y, Wang W, Xu M, Wang T, Lu J, Xu Y, et al. Diabetes genetic risk score modifies effect of bisphenol A exposure on deterioration in glucose metabolism. J Clin Endocrinol Metab. 2016;101(1):143-50. 